\title{
Should revascularisation be recommended for atherosclerotic renal artery stenosis?
}

\author{
V Jha \\ Additional Professor of Nephrology, Postgraduate Institute of Medical Education and Research, Chandigarh, India
}

TITLE Revascularization versus medical therapy for renal-artery stenosis

AUTHORS ASTRAL Investigators

JOURNAL N Engl J Med 2009; 361:1953-62

DECLARATION OF INTERESTS Professor Jha has declared no conflict of interests.

\author{
Correspondence to V Jha, \\ Postgraduate Institute of Medical \\ Education and Research, \\ Chandigarh 160 012, India \\ tel. +9 I I72 2756733 \\ e-mail vjha@pginephro.org
}

\section{SUMMARY}

This multicentric, multinational, randomised controlled trial (RCT) was aimed to see if percutaneous revascularisation of the renal arteries in atherosclerotic renovascular disease provided clinical benefits. A total of 806 patients in whom the benefits of revascularisation were thought to be uncertain by their treating physicians were randomised to the medical therapy or the revascularisation plus medical therapy arms. Randomisation was stratified according to serum creatinine levels, Cockcroft-Gault glomerular filtration rate (GFR), severity of renal-artery stenosis, kidney length and rate of progression of renal impairment before entry. The primary outcome was the rate of change of renal function as measured by the reciprocal of the serum creatinine. Blood pressure, time to renal and major cardiovascular events and mortality were additional outcome variables.

Of the 403 patients randomised to the revascularisation arm, 335 underwent the procedure; $95 \%$ had a stent placed. The technical success was $95 \%$. The crossover rate to revascularisation in the medical therapy group was $6 \%$. Patients were followed up for a median period of 34 months. The rate of change of the slope of the inverse of serum creatinine during follow-up was not different in the two groups $\left(-0.07 \times 10^{-3}\right.$ and $-0.13 \times 10^{-3} \mathrm{l} / \mu \mathrm{mol} /$ year in the revascularisation and medical therapy groups respectively). There was a difference favouring revascularisation of $0.06 \times 10^{-3} \mathrm{l} / \mu \mathrm{mol} /$ year $(95 \%$ confidence interval, -0.002 $0.13 ; p=0.06$ ). There was no difference in the change in serum creatinine, systolic blood pressure or rates of renal events, major cardiovascular events and death. The medical therapy group required more antihypertensive agents at one year $(2.97$ vs $2.77, p=0.03)$. There were no differences in the outcome in any of the predefined subgroups, including those with high-grade or bilateral stenosis or impaired or decreasing kidney function at entry. Thirtyone patients developed complications within 24 hours of the procedure, and another 55 patients in the next 30 days. These were deemed to be serious in 23 patients, including two deaths and three amputations. The authors concluded that there was no evidence of a worthwhile clinical benefit from revascularisation in patients with atherosclerotic renovascular disease.

\section{OPINION}

Atherosclerotic renal artery stenosis (RAS) is the most common correctable cause of hypertension. When bilateral or present in a solitary functioning kidney, RAS can cause a progressive loss of renal function. Ischaemic nephropathy is listed as the cause of end-stage renal disease in about 12-20\% of patients.' Atherosclerotic RAS increases the risk for cardiac events (pulmonary oedema, refractory heart failure or acute coronary ischaemia), stroke and peripheral vascular disease. Renal insufficiency in patients with RAS is associated with markedly decreased survival. In a study of patients undergoing coronary angiography, RAS was a stronger independent predictor of all-cause mortality than congestive heart failure, left ventricular ejection fraction or the level of renal dysfunction. ${ }^{2}$

The benefits of revascularisation in RAS may seem selfevident. 'Drive-by' angiographies are frequently performed during coronary or peripheral angiograms. Incidental RAS is discovered in 15-35\%, and prompts percutaneous renal angioplasty and stenting in many. ${ }^{3}$ The American Heart Association guidelines ${ }^{4}$ on this procedure have been criticised, ${ }^{5}$ not least because revascularisation is associated with complications in a significant proportion of patients.

Whether revascularisation provides tangible clinical benefits remains unclear. Percutaneous renal angioplasty alone is technically unsatisfactory for the ostial lesions of atherosclerotic RAS, and has high restenosis rates. Percutaneous renal angioplasty trials have failed to show an effect on blood pressure or kidney function. A pooled meta-analysis ${ }^{6}$ suggested a modest improvement in blood pressure with revascularisation. These studies, however, were bedevilled by methodological issues, including inadequate sample size and imprecise patient selection criteria. A significant proportion crossed over from medical therapy to angioplasty, reducing the validity of the results.

Following the introduction of stents, the technical success of revascularisation improved and the risk of restenosis came down. Observational data and non-randomised 
studies showed improved blood pressure control and less worsening of renal function following stent placement. ${ }^{7-11}$

On this background, the Angioplasty and STenting for Renal Artery Lesions (ASTRAL) study results are significant.This was a well-conducted RCT with predefined outcomes. Unlike previous trials, not many patients crossed over from the conservative to the intervention arm. However, ASTRAL turned out to be another addition to the list of negative studies in the nephrology field.

Do the results sound a death knell for revascularisation for atherosclerotic RAS? Perhaps not, since concerns remain that make this study less than definitive. The inclusion criteria are vague, to say the least. Patients were required to have 'stenosis in at least one renal artery that was considered potentially suitable for endovascular revascularisation' and 'if the patient's doctor was uncertain that the patient would definitely have a worthwhile clinical benefit from revascularisation'. This necessarily excluded patients who in the doctors' opinion would benefit from the procedure and says little about the characteristics of the studied patients.

A closer inspection of the data reveals the crucial fact that 32 I out of the $403(80 \%)$ patients in the revascularisation group did not have stenosis that would be considered clinically significant according to current knowledge. This means that the likelihood of benefit in them was low in any case. In fact, only 163 patients in both groups had highgrade $(\geq 70 \%)$ RAS involving both renal arteries or the artery supplying a single functioning kidney. Considering that the primary outcome was slowing of the progression of renal dysfunction, the subgroup of cases with significant disease is clearly underpowered to detect a benefit. The observed rate of decline of renal function in the medical therapy group was much slower than anticipated by the authors. The authors interpret this to mean that good medical therapy is useful in slowing progression. However, it is equally likely that the slow progression was because of low-grade disease.

Another couple of points need a mention. Distal protection devices, which can reduce some of the complications, were not used in this study. Finally, the trial did not have enough power to detect a difference in cardiovascular event rates.

Another recently published RCT, the STenting in renal dysfunction caused by Atherosclerotic Renal artery (STAR) trial, ${ }^{12}$ randomised 140 patients with ostial RAS involving $>50 \%$ of the luminal diameter and CockroftGault GFR of $<80 \mathrm{ml} / \mathrm{min} / \mathrm{l} .73 \mathrm{~m}^{2}$. This trial also did not find stenting to be better than medical therapy for the prevention of loss of kidney function, but the study was underpowered to detect a difference in GFR.

The Cardiovascular Outcomes with RenalAtherosclerotic Lesions (CORAL) study is currently under way. ${ }^{13}$ The study entry criteria are stenosis of $\geq 60 \%$ with a systolic pressure gradient of at least $20 \mathrm{mmHg}$, or $\geq 80 \%$ without gradient. The study endpoint is event-free survival from a composite of several cardiovascular and renal events. Distal protection devices will be used at the time of stenting. Preliminary study results are expected in $201 \mathrm{I}$.

In summary, the result of the ASTRAL trial can be interpreted to mean that the best treatment of RAS remains uncertain unless we find a good means of identifying patients suitable for the procedure, preferably in a noninvasive way. Brain natriuretic peptide could be one such marker. ${ }^{3}$ Future trial protocols need to include these.

\section{REFERENCES}

I Fatica RA, Port FK, Young EW. Incidence trends and mortality in endstage renal disease attributed to renovascular disease in the United States. Am J Kidney Dis 200 I; 37:I I84-90. doi:I0.1053/ajkd.200I.2452I

2 Conlon PJ, Athirakul K, Kovalik E, et al. Survival in renal vascular disease.J Am Soc Nephrol 1998; 9:252-6.

3 White CJ. Management of renal artery stenosis: the case for intervention, defending current guidelines, and screening (driveby) renal angiography at the time of catheterization. Prog Cardiovasc Dis 2009; 52:229-37. doi:10.1016/j.pcad.2009.09.006

4 White CJ, Jaff MR, Haskal ZJ et al. Indications for renal arteriography at the time of coronary arteriography: a science advisory from the American Heart Association Committee on Diagnostic and Interventional Cardiac Catheterization, Council on Clinical Cardiology, and the Councils on Cardiovascular Radiology and Intervention and on Kidney in Cardiovascular Disease. Circulation 2006; I |4:I892-5. doi: I0.I I6I/CIRCULATIONAHA.I06.I78777

5 Dear JW, Padfield PL, Webb DJ. New guidelines for drive-by renal arteriography may lead to an unjustifiable increase in percutaneous intervention. Heart 2007; 93: I 528-32. doi:I0.I I36/hrt.2007.II7275

6 Nordmann AJ, Logan AG. Balloon angioplasty versus medical therapy for hypertensive patients with renal artery obstruction. Cochrane Database Syst Rev 2003; 3:CD002944.

7 Harden PN, MacLeod MJ, Rodger RS et al. Effect of renal-artery stenting on progression of renovascular renal failure. Lancet 1997; 349:I I33-6. doi: I0.1016/S0|40-6736(96) I0093-3

8 Watson PS, Hadjipetrou P, Cox SV et al. Effect of renal artery stenting on renal function and size in patients with atherosclerotic renovascular disease. Circulation 2000; 102:167I-7.

9 Burket MW, Cooper CJ, Kennedy DJ et al. Renal artery angioplasty and stent placement: predictors of a favorable outcome. Am Heart J 2000; I39:64-7I. doi:10.1016/S0002-8703(00)90310-7

10 Pizzolo F, Mansueto G, Minniti S et al. Renovascular disease: effect of ACE gene deletion polymorphism and endovascular revascularization. JVasc Surg 2004; 39:140-7. doi:I0.1016/S0741-52 I4(03)0I018-8

II Rocha-Singh K, Jaff MR, Rosenfield K. Evaluation of the safety and effectiveness of renal artery stenting after unsuccessful balloon angioplasty: the ASPIRE-2 study. J Am Coll Cardiol 2005; 46: 776-83. doi:10.1016/j.jacc.2004.11.073

12 Bax L, Woittiez AJ, Kouwenberg HJ et al. Stent placement in patients with atherosclerotic renal artery stenosis and impaired renal function: a randomized trial. Ann Intern Med 2009; I50: 840-8, WI50-I.

I3 Murphy TP, Cooper CJ, Dworkin LD et al. The Cardiovascular Outcomes with Renal Atherosclerotic Lesions (CORAL) study: rationale and methods. J Vasc Interv Radiol 2005; 16:I 295-300. 